\title{
Home Management of Childhood Asthma Exacerbations
}

Telford Y. Yeung - Christopher Ewing - Anna Malanowska •

Peter Zuberbuhler · Michelle Balcom · Janny Liu · Israel Amirav

To view enhanced content go to www.pulmonarytherapy-open.com Received: July 18, 2018 / Published online: August 31, 2018

(C) The Author(s) 2018

\section{ABSTRACT}

Introduction: Effective home management of childhood asthma by caregivers requires education along with a written asthma action plan (AAP), which should outline clear instructions for treatment during exacerbations. However, a large number of asthma exacerbations continue to be managed in the emergency department (ED) and in hospitals, particularly in Canada. The objective of this study was to assess caregiver management of acute asthma at home following the 2015 Global Initiative for Asthma (GINA) guidelines and to identify factors that

Enhanced Digital Features To view enhanced digital features for this article go to https://doi.org/10.6084/ m9.figshare.6984551.

Electronic supplementary material The online version of this article (https://doi.org/10.1007/s41030018-0061-y) contains supplementary material, which is available to authorized users.

T. Y. Yeung $\cdot$ A. Malanowska $\cdot$ M. Balcom $\cdot$ J. Liu I. Amirav ( $\square)$

Stollery Pediatric Pulmonary Clinic, 8440112 Street, Edmonton, AB, Canada

e-mail: amirav@ualberta.ca

T. Y. Yeung · I. Amirav

Edmonton Clinic Health Academy, University of

Alberta, 1140587 Avenue, Edmonton, AB, Canada

C. Ewing · P. Zuberbuhler

Baker Pediatric Clinic, Edmonton, AB, Canada may be associated with deviations from these guidelines.

Methods: 122 caregivers of children, aged 317 years, with physician diagnosed asthma, completed a paper-based questionnaire. Correct caregiver management (defined according to the GINA guidelines) of acute asthma as well as their use of an AAP were assessed.

Results: Out of all caregivers, $74.6 \%$ incorrectly treated their child's asthma exacerbation in a home setting. Among those who used an AAP, we observed significantly more ED visits $(0.9 \pm 1.2$ versus $0.5 \pm 0.9, p=0.04)$ and hospitalizations $(0.2 \pm 0.4$ versus $0.0 \pm 0.0$, $p=0.02$ ) when compared to non-AAP users in the past 1 year.

Conclusions: Caregivers of children with asthma in Canada may still lack skills for proper home management of asthma exacerbations. We found a higher number of ED visits and hospitalizations in those using an AAP compared to those who did not use an AAP. These data suggest that current AAPs may not be sufficient for home asthma management.

Keywords: Asthma; Asthma control; Asthma management; Action plan; Children

\section{INTRODUCTION}

Asthma is among the most common chronic diseases in children [1]. The lifetime prevalence 
of asthma in Canadian children has been estimated at $11-16 \%$ [2]. Children are frequently at risk for asthma exacerbations from viral infections, physical activity, as well as exposure to allergens. Thus, having adequate knowledge to manage asthma at home is critical to avoid exacerbations and to initiate treatment properly [3]. The asthma action plan (AAP) is a document provided to caregivers that should outline what medication is needed every day, what steps to take for an asthma exacerbation, and when to call a doctor or to seek emergency treatment. Both national and international guidelines recommend self-management approaches such as the use of AAP for home management of asthma [4, 5]. In addition, use of an AAP results in fewer acute asthma ED visits, less nighttime awakenings, fewer missed school days, and better asthma symptom scores [6].

Despite the general acceptance of these approaches $[7,8]$, there is still substantial use of the emergency department (ED) and the hospital to manage acute exacerbations of asthma. Approximately $10 \%$ of pediatric emergency visits for asthma in Alberta resulted in hospital admission [9]; in addition, the rate of visits to the ED in Alberta (20/1000) exceeds that of their peers in the US $(9 / 1000)[9,10]$. Several caregiver factors (lack of knowledge, skills, or confidence to provide care for their child during an asthma exacerbation) may contribute to hospital and emergency department use [11]. In addition, concerns have been raised that despite having an AAP, the instructions contained in Canadian AAPs may be vague and confusing for parents [12]. Nevertheless, very little data is available to assess Canadian caregivers' knowledge and their actions to manage acute asthma at home. We hypothesize that the increased use of ED and hospital by children with asthma in Canada may be related to inappropriate home management, along with not following or not using the AAP.

The objective of this study was to assess the home management of acute asthma by caregivers (as per the 2015 GINA asthma guidelines), and to identify potential factors that may be associated with incorrect management. Specifically, this study sought to answer whether caregivers, (1) followed the instructions provided in their AAP (if provided), (2) were confident in their knowledge and skills to recognize and manage an acute asthma exacerbation, and (3) knew what actions to take for home management of acute asthma.

\section{METHODS}

\section{Participants}

We collected data from caregivers of children who were known to suffer from asthma. Inclusion criteria included parents/caregivers of children that were: (1) between 3 and 17 years of age, (2) with a previous physician made diagnosis of asthma. Children with new consultations for wheezing or cough were not included without a prior diagnosis of asthma. Children of less than 3 years of age were also excluded in order to avoid misclassification in that age group [13]. Participants were consecutively enrolled from outpatient visits and the patient health care identifier was recorded to ensure that no participants were recruited more than once. We sent a letter that explained the purpose of the study to caregivers by an e-mail 1 week before their child's regular clinic visit. Caregivers who agreed to participate in this study verbally consented. Children were recruited from the Stollery Children's Hospital outpatient respiratory clinic and the Baker Clinic pediatric department in Edmonton, Canada between September 1, 2015 and November 17, 2017.

\section{Compliance with Ethics Standards}

All procedures performed in studies involving human participants were in accordance with the ethical standards of the institutional and/or national research committee and with the 1964 Helsinki Declaration and its later amendments or comparable ethical standards. Informed consent was obtained from all individual participants included in the study. The study was approved by the University of Alberta ethics committee (Human REB Pro00064186). 


\section{Questionnaire}

At the clinic visit, the participants completed a 15-min questionnaire that evaluated the caregivers' knowledge of asthma along with their management steps during acute asthma exacerbations and their confidence in self-management of these episodes (Supplementary Figure 1). This questionnaire was developed based on questions employed in a previous study on home management of asthma in children [15]. Data on demographics, use of an AAP, and a validated asthma control questionnaire (ACQ, [14]) assessing degree of asthma control were also collected. The ACQ consists of six questions about key symptoms (dyspnea and wheezing), daytime and nighttime symptoms, activity limitation, and rescue bronchodilator (Supplementary Figure 1). Each item is scored on a seven-point scale ranging from 0 to 6 . The final score is obtained by averaging the total scores for the six items. Higher scores indicate worse asthma control.

\section{Criteria for Correct and Incorrect Home Management}

In the questionnaire, parents were presented with a theoretical scenario of an acute asthma exacerbation at home and were asked to describe management actions. Both initial step and subsequent steps were recorded (Supplementary Figure 1). Correct management of an acute exacerbation was defined according to the recent 2015 Global Initiative for Asthma (GINA) guidelines [5]: The caregiver should initiate treatment with two puffs of inhaled short acting beta agonist (SABA, salbutamol $200 \mu \mathrm{g}$ or equivalent), given one puff at a time via a spacer device with or without a facemask. This may be repeated a further two times at 20-min intervals, if needed.

Incorrect management was defined by any management of asthma that did not follow the initial two puffs of inhaled short acting beta agonist given one puff at a time, followed if needed by two repeats at 20-min intervals as required.
We analyzed for differences in the proportion of "correct" and "incorrect" home management of asthma. We also analyzed for factors which may be associated with "correct" and "incorrect" home management, including previous hospitalizations and number of emergency department visits in the past year, knowledge of asthma, confidence in selfmanaging asthma attacks, degree of asthma control, and use of an AAP.

\section{Statistical Analysis}

For statistical analyses, we used a two-tailed Fischer's test of proportions, a two-tailed Student's $t$ test of means and a Pearson correlation. We used an alpha level of 0.05 and a power of 0.80 to establish the appropriate sample size. Based on a similar study [15], in which the proportion of correctly managed to incorrectly managed was $1: 2$, we calculated the sample size required for our study based on this ratio to be 114. Data collected were entered into a Microsoft Excel 2016 database and were analyzed using GraphPad Prism 6.0/STATA 11 according to the criteria defined for proper home management of asthma. All values reported are expressed as mean \pm standard deviation.

\section{RESULTS}

A total of 127 caregivers were given surveys to complete and 122 questionnaires were completed (Table 1). Five caregivers did not want to participate and they were not included. The average score for the ACQ was $0.7 \pm 0.8$ (out of a total score of 6), indicating that this was a sample of well-controlled children with asthma. Of the participants, $62.3 \%$ experienced an asthma exacerbation in the previous year, which resulted in an average of $1.0 \pm 1.6$ emergency visits over the same period (Table 1). Nearly two-thirds of patients used an AAP $(65.5 \%)$. However, only $51.6 \%$ of caregivers chose the correct initial choice of treatment for asthma exacerbation as per the GINA 2015 guidelines (Table 2). Subsequently only $46.7 \%$ of caregivers were able to identify the correct follow-up management step (Table 2). In total, 
Table 1 Demographics

Total number of surveys distributed 127

Total number of participants completing surveys 122

Average age of participants (years)

$8.9 \pm 3.6$

Proportion older than 12 years (\%)

15.6

Sex (\% female)

Average age of diagnosis of asthma (years)

$4.5 \pm 3.0$

Percentage of participants with asthma exacerbation over the last year

Average number of emergency visits for patients with asthma exacerbations over the last year

$1.0 \pm 1.6$

Average number of hospitalizations for patients with asthma exacerbations over the last year

$0.1 \pm 0.4$

Patients with AAP (\%)

70.5

Patients who use AAP (\%)

Caregivers who understand AAP (\%)

67.2

only $25.4 \%$ of caregivers were able to correctly identify both the initial and follow-up steps for an asthma exacerbation; and $74.6 \%$ of caregivers incorrectly identified the initial and follow-up steps of asthma exacerbation management. We assessed for type of rescue inhaler use and we found that most families used salbutamol (92\%) and only a minority used bricanyl (4\%). A minority of patients used combined inhaled steroids and long-acting beta agonists (4\%).

Correct management of asthma exacerbations was not associated with fewer ED visits (correct: $0.8 \pm 1.1$ visits versus incorrect: $0.8 \pm 1.2$ visits, $p=0.93$ ) or hospitalizations (correct: $0.1 \pm 0.3$ hospitalization versus incorrect: $0.1 \pm 0.4$ hospitalizations, $p=0.76$ ) over the past year (Table 3 ). We also found no association between correct management of asthma exacerbations and AAP use, asthma knowledge, asthma control scores (ACQ scores) or self-confidence in asthma management (Table 3). No correlation was found between ACQ scores and the number of ED visits $\left(R^{2}=0.087\right)$ or the number of hospitalizations for asthma exacerbations $\left(R^{2}=0.002\right)$.

When we stratified the population into use of the asthma action plan, we found that caregivers who used an asthma action plan (AAP) visited the emergency significantly more often
(AAP use: $1.0 \pm 1.3$ versus no AAP use: $0.5 \pm 0.9, \quad p=0.04)$ and had significantly increased hospital admissions (AAP use: $0.2 \pm 0.4$ versus no AAP use: $0.0 \pm 0.0$, $p=0.02)$ compared to those who did not use an AAP (Table 4). We found no difference in asthma knowledge scores or asthma control scores. Confidence in initiating treatment for asthma exacerbation was greater in those who used an AAP (AAP use: $8.9 \pm 1.4$ versus no AAP use: $8.3 \pm 2.0, p=0.04$ ); however, confidence in recognizing an asthma exacerbation and stopping treatment was no different between the two groups (Table 4).

\section{DISCUSSION}

In Canada, a substantial number of emergency department visits and hospital admissions for children have been related to asthma exacerbations [9]. Recent evidence shows that factors such as caregivers' lack of knowledge, skills, and confidence contribute to improper management of asthma exacerbations at home [16]. We believe each of these factors is also linked to the increased ED visits and hospital admissions. As previously reported [15], the present study also found that only a minority of caregivers appropriately managed asthma exacerbations at 
Table 2 Home management decisions for therapy of asthma exacerbation

\begin{tabular}{llc}
\hline & Responses & Choosing (\%) \\
\hline Initial choice of therapy for home management of & Call MD & 1.6 \\
asthma exacerbations & Ventolin nebulizer & 4.9 \\
& Ventolin, 1 puff & 20.5 \\
& Ventolin, 2 puffs one at a time ${ }^{\mathrm{a}}$ & 51.6 \\
& Ventolin, 2 puffs together & 7.4 \\
& Ventolin, 3 puffs one at a time & 1.6 \\
& Ventolin, 3 puffs together & 0.0 \\
& Ventolin, 4 puffs one at a time & 7.4 \\
& Ventolin, 4 puffs together & 1.6 \\
Immediate follow-up therapy for home management & Call MD & 11.4 \\
of asthma exacerbations & Repeat treatment in 20-min ${ }^{\mathrm{a}}$ & 46.7 \\
& Repeat treatment in 2 h & 20.5 \\
& Repeat treatment in 4h & 13.1 \\
& Go to emergency & 4.9 \\
\hline
\end{tabular}

$3.3 \%$ of participants did not provide a response

${ }^{a}$ Represents those who chose proper management steps defined in the methods section as based on the GINA 2015 guidelines. A total of $25.4 \%$ of individuals chose proper management, which required the correct steps for both initial and immediate therapy for asthma exacerbation

Table 3 Clinical outcomes with proper management of asthma exacerbations

\begin{tabular}{llll}
\hline & Correct & Incorrect & $\boldsymbol{p}$ value \\
\hline Emergency department visits over the last year & $0.8 \pm 1.1$ & $0.7 \pm 1.2$ & 0.93 \\
Hospitalizations over the last year & $0.1 \pm 0.4$ & $0.1 \pm 0.3$ & 0.76 \\
Asthma action plan use & $18 / 31(58.1 \%)$ & $63 / 91(69.2 \%)$ & 0.28 \\
Asthma knowledge score & $10.5 \pm 1.6$ & $10.0 \pm 1.5$ & 0.13 \\
Asthma control score & $0.8 \pm 0.9$ & $0.7 \pm 0.7$ & 0.57 \\
Confidence scores & & & \\
Recognize asthma exacerbation & $8.3 \pm 1.7$ & $8.3 \pm 1.8$ & 0.97 \\
Initiate treatment of asthma exacerbation & $9.2 \pm 1.2$ & $8.6 \pm 1.7$ & 0.06 \\
Stop treatment of asthma exacerbation & $7.2 \pm 2.8$ & $6.8 \pm 2.7$ & 0.45 \\
\hline
\end{tabular}

home. Our data showed that proper management of asthma exacerbations was not associated with a decrease in $\mathrm{ED}$ visits $(p=0.93)$ or hospitalizations $(p=0.76)$ over the past year.
We also found no association between correct home management of asthma exacerbations and AAP use, asthma knowledge, asthma control scores, or confidence in asthma 
Table 4 Role of asthma action plan on acute asthma management at home

\begin{tabular}{llll}
\hline & AAP use & No AAP use & $\boldsymbol{p}$ value \\
\hline Improper management & $63 / 80(78.7 \%)$ & $24 / 42(64.3 \%)$ & 0.17 \\
ED visits & $0.9 \pm 1.2$ & $0.5 \pm 0.9$ & 0.04 \\
Hospitalizations & $0.2 \pm 0.4$ & $0.0 \pm 0.0$ & 0.02 \\
Asthma knowledge score & $10.0 \pm 1.6$ & $10.4 \pm 1.4$ & 0.21 \\
Asthma control score & $0.7 \pm 0.7$ & $0.7 \pm 0.8$ & 0.75 \\
Confidence scores & & $7.9 \pm 1.9$ & 0.06 \\
Recognize asthma exacerbation & $8.5 \pm 1.6$ & $8.3 \pm 2.0$ & 0.04 \\
Initiate treatment of asthma exacerbation & $8.9 \pm 1.4$ & $7.3 \pm 2.6$ & 0.23 \\
Stop treatment of asthma exacerbation & $6.7 \pm 2.8$ & & \\
\hline
\end{tabular}

management. These data are unexpected but our data show that caregivers have less confidence in continuing asthma management at home. Caregivers are more certain with initiating rather than continuing asthma management and subsequently present to the emergency for further management.

Surprisingly, we showed that despite using an asthma action plan (AAP) and having greater confidence in initiating home treatment for asthma exacerbations, caregivers had significantly more emergency department use $(p=0.04)$ and increased hospitalizations $(p=0.02)$ compared to those who did not have an AAP. Our data illustrate an association instead of a causal relationship; nevertheless, these data suggest that caregivers may feel comfortable starting treatment at home but are uncomfortable or lack knowledge/skills for managing asthma afterwards and thus may easily resort to the ED. Taken together, these data suggest that asthma ED visits and hospitalizations may not necessarily be caused by failing to follow the AAP instructions but rather by a lack of clear instructions regarding treatment after initiating treatment. Patients want clear and specific instructions in their AAPs to manage acute exacerbations at home [17]. The AAP used in our patient's population was the ICAN AAP (http://www.ucalgary.ca/ icancontrolasthma). This AAP is a non-generic plan and is customized to a patient's specific situation. However, like other Canadian AAPs, the ICAN AAP combines instructions for the management of acute exacerbations with advice for the general management of asthma, which can be confusing [18]. Adding the concept of asthma control to the Canadian AAP and thus placing the responsibility of assessing control on caregivers may cause considerable confusion in caregivers during acute exacerbations [12].

Guidelines inform AAPs. In contrast to current Canadian guidelines, other national and international guidelines and their AAPs provide very specific instructions for home treatment of acute exacerbations. The GINA guidelines [5] for initial home management of asthma exacerbations in children 5 years and younger are to use "two puffs of short-acting beta agonist and repeat a further two times at 20-min intervals, if needed" [5]. Similar precise actions are recommended by the British Thoracic Society (where a public health system similar to Canada exists) and by Australian Guidelines [19, 20]. In contrast, the Canadian AAPs focus on "control" issues and may not achieve the intended purpose of an action plan, which is to provide guidance for treating acute transient exacerbations $[12,18]$. A review of caregiver's understanding and their use of AAP for home management combined with clear AAP instructions [21] is crucial to ensure adequate knowledge and skill for home management.

While the level of asthma control in this study was relatively high (mean ACQ of $0.7 \pm 0.8$ ), we observed a relatively high average 
number of ED and hospital use. This discrepancy demonstrates the limitations of the ACQ as the test is subjective and reflects the asthma control in the short term, which may have overestimated true asthma control [22]. Thus, we acknowledge that the caregivers in our study who had an AAP may also have children with more severe asthma. Another plausible explanation is that in the Canadian health-care system, there is unlimited use of ED/hospital. In a similar way that the AAPs did not provide the patients clear instructions about home management, parents may rely on the ED rather than attempt home self-management. In that regard, it is interesting to read the following citation from a focus group of pediatricians who discussed management of acute asthma at home: "Why can't they go to emergency? Isn't that what the emergency room is for? At night, if the kid's wheezing at 3:00 in the morning, that's probably where they should be" [23]. This statement indicates how confusing this issue has become, even for physicians.

Interestingly, recent studies have suggested that AAPs may not provide additional benefit to asthma education and regular follow up for asthma outcomes [24, 25]. In fact, written asthma action plans may have discouraged caregivers from seeking medical attention until hospitalization was required [24]. In addition to our results, these findings demonstrate that current AAPs can mislead caregivers regarding management of asthma at home.

\section{Limitations}

We acknowledge that our study is single-centered, and that management of asthma may differ between various geographic regions of Canada. Regarding data collection, our study is at risk for recall bias as caregivers were asked to report data from the previous year. Although the parents were asked: "has a doctor/nurse ever given you an asthma action plan for your child?", the date when caregivers were given an $\mathrm{AAP}$, and the date of ED visits as well as hospitalizations (in the last year) were not recorded. The potential confounder is that some caregivers may have been provided an AAP after their ED visit or hospitalization. In these cases, the ED visits served to promote referrals to specialists who then provided an AAP instead of the AAP being associated with increased ED visits and hospitalizations.

\section{Conclusions and Future Directions}

Efficacious management of asthma and acute exacerbations requires considerable and repeated asthma education with written AAPs that are simple and explicit. In this study, we found that caregivers who used the Canadian AAP in our pediatric hospital's catchment area had a significantly higher number of ED visits and hospitalizations but no difference in "asthma knowledge". These results suggest that instructions in the AAP may not provide sufficiently clear instructions for management of acute asthma exacerbations at home. We suggest that the Canadian AAPs be revisited in order to ensure that we are providing our patients with clear and understandable guidelines that focus on self-management of asthma exacerbations. We believe that providing explicit instructions on management of acute exacerbations to caregivers of children with asthma can improve their confidence and management of asthma at home.

\section{ACKNOWLEDGEMENTS}

This study was presented at Pediatric Academic Society 2018 in Toronto, Canada and travel was supported by the Women and Children's Health Research Institute (WCHRI) Travel grant. The primary author was awarded a WCHRI Resident Trainee Grant (TYY). We would like to thank Dr. Michael Newhouse, MD, MSc, from McMaster University for his expertise and review of this manuscript. We would also like to thank all participants of this study.

Funding. No funding or sponsorship was received for this study or publication of this article. 
Authorship. All named authors meet the International Committee of Medical Journal Editors (ICMJE) criteria for authorship for this article, take responsibility for the integrity of the work as a whole, and have given their approval for this version to be published.

Disclosures. Telford Y Yeung, Christopher Ewing, Anna Malanowska, Michelle Balcom, and Janny Liu have nothing to disclose. The corresponding author, Israel Amirav, is a member of the editorial board for Pulmonary Therapy. He has also published a recent commentary on asthma action plans in December 2017.

Compliance with Ethics Guidelines. All procedures performed in studies involving human participants were in accordance with the ethical standards of the institutional and/or national research committee and with the 1964 Helsinki Declaration and its later amendments or comparable ethical standards. Informed consent was obtained from all individual participants included in the study.

Data Availability. The dataset analyzed during the current study is available from the corresponding author on reasonable request.

Open Access. This article is distributed under the terms of the Creative Commons Attribution-NonCommercial 4.0 International License (http://creativecommons.org/licenses/ by-nc/4.0/), which permits any noncommercial use, distribution, and reproduction in any medium, provided you give appropriate credit to the original author(s) and the source, provide a link to the Creative Commons license, and indicate if changes were made.

\section{REFERENCES}

1. Garner R, Kohen D. Changes in the prevalence of asthma among Canadian children. Health Rep. $2008 ; 19(2): 45-50$.

2. To T, Dell S, Tassoudji M, Wang C. Health outcomes in low-income children with current asthma in Canada. Chronic Dis Can. 2009;29(2):49-55.
3. Gibson PG, Powell H, Wilson A, et al. Self-management education and regular practitioner review for adults with asthma. Cochrane Database Syst Rev. 2003;1:CD001117.

4. Lougheed MD, Lemiere C, Ducharme FM, Licskai C, Dell SD, Rowe BH, Fitzgerald M, Leigh R, Watson W, Boulet LP. Canadian Thoracic Society asthma clinical assembly. Canadian Thoracic Society 2012 guideline update: diagnosis and management of asthma in preschoolers, children and adults. Can Respir J. 2012;19(2):127-64.

5. Global Strategy for Asthma Management and Prevention, Global Initiative for Asthma (GINA) 2015. http://www.ginasthma.org/. Accessed Dec 2017.

6. Zemek RL, Bhogal SK, Ducharme FM. Systematic review of randomized controlled trials examining written action plans in children: What is the plan? Arch Pediatr Adolesc Med. 2008;162:157-63.

7. Larson A, Ward J, Ross L, Whyatt D, Weatherson M, Landau L. Impact of structured education and selfmanagement on rural asthma outcomes. Aust Fam Physician. 2010;39:141-4.

8. Ortiz-Alvarez O, Mikrogianakis A. Managing the pediatric patient with an acute asthma exacerbation. Paediatr Child Health. 2012;17:251-5.

9. Rosychuk RJ, Voaklander DC, Klassen TP, Senthilselvan A, Marrie TJ, Rowe BH. Asthma presentations by children to emergency departments in a Canadian province: A population-based study. Pediatr Pulmonol. 2010;45(10):985-92.

10. Nath JB, Hsia RY. Children's emergency department use for asthma 2001-2010. Acad Pediatr. 2015; 15:225-30.

11. Deis JN, Spiro DM, Jenkins CA, Buckles TL, Arnold $\mathrm{DH}$. Parental knowledge and use of preventive asthma care measures in two pediatric emergency departments. J Asthma. 2010;47(5):551-6.

12. McGillivray ME, Flavin MP. Canadian pediatric asthma action plans and their correlation with current consensus guidelines. Pediatr Child Health. 2014;19(7):362-6.

13. Ducharme F, Dell SD, Radhakrishnan D, Grad RM, Wade TA, et al. Diagnosis and management of asthma is preschoolers: A Canadian Thoracic Society and Canadian Pediatric Society position paper. Can Respir J. 2015;22:135-43.

14. Nguyen JM, Holbrook JT, Wei CY, Gerald LB, Teague WG, Wise RA. Validation and psychometric properties of the asthma control questionnaire 
among children. J Allergy Clin Immunol. 2014;133:91-7.

15. Clayton K, Monroe K, Magruder T, King W, Harrington $\mathrm{K}$. Inappropriate home albuterol use during an acute asthma exacerbation. Ann Allergy Asthma Immunol. 2012;109:416-9.

16. Al-Muhsen S, Horaneih N, Dulgom S, Al Aseri Z, Vasquez-Tello A, Halwani R, Al-Jahdali H. Poor asthma education and medication compliance are associated with increase emergency department visits by asthmatic children. Ann Thorac Med. 2015;10:123-31.

17. Dinakar C, Van Osdol TJ, Wible K. How frequent are asthma exacerbations in a pediatric primary care setting and do written asthma action plans help in their management? J Asthma. 2004;41:807-12.

18. Amirav I, Newhouse MT. Asthma control and action plans. Eur Respir J. 2017;50(6):1701640.

19. British Thoracic Society/Scottish Intercollegiate Guidelines Network. British guideline on the management of asthma: a national clinical guideline. http://www.brit-thoracic.org.uk/document-library/ clinical-information/asthma/btssign-asthma-guide line-2016/. Accessed Dec 2017.

20. Clinical Practice Guidelines. The Royal Children's Hospital Melbourne. http://www.rch.org.au/
clinicalguide/forms/Asthma_Action_Plan/. Accessed 12 Aug 2017.

21. Yin H, Gupta R, Tomopoulos S, et al. Readability, suitability and characteristics of asthma action plans: examination of factors that may impair understanding. Pediatrics. 2013;131:1-11. https:// doi.org/10.1542/peds.2012-0612.

22. Price $D$, Fletcher $M$, van der Molen T. Asthma control and management in 8000 European patients: the REcognise asthma and LInk to symptoms and experience (REALISE) survey. NPJ Prim Care Respir Med. 2014;24:14009.

23. Smith N, Smith A, Wang A, Shaw K, Groeneweg G, Carleton B, et al. Physician and parent barriers to the use of oral corticosteroids for the prevention of paediatric URTI-induced acute asthma exacerbations at home. Paediatr Child Health. 2017;22(4):190-4. https://doi.org/10.1093/pch/ p047.

24. Kelso JM. Do written asthma action plans improve outcomes? Pediatr Allergy Immunol Pulmonol. 2016;29:2-5.

25. Sheares BJ, Mellins RB, Dimango E, Serebrisky D, Zhang Y, Bye MR, et al. Do patients of subspecialist physicians benefit from written asthma action plans? Am J Respir Crit Care Med. 2015;191(12):1374-83. 